\title{
Evaluación de la competencia transversal "Responsabilidad ética, medioambiental y profesional” a través de una e-rúbrica en el laboratorio.
}

Bañuls $M^{a}$ Joséa $^{a}$, López-Gresa $M^{a}$ Pilarb, Cebolla-Cornejo Jaime ${ }^{b}$, Díez $\mathbf{M}^{\mathrm{a}}$ Joséb$^{\mathrm{b}}$, Esteras Cristina ${ }^{b}$, Ferriol Maríac, González-Martínez Miguel Angela, Leiva-Brondo

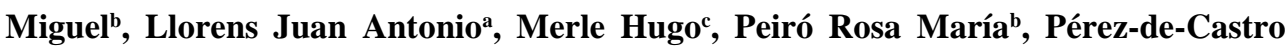
Ana Maríab, Picó Belén ${ }^{b}$, Lisón Purificación'.

aDepartemento de Química, 'Departamento de Biotecnología y ${ }^{\mathrm{c} D e p a r t a m e n t o ~ d e ~ E c o s i s t e m a s ~}$ Agroforestales

\begin{abstract}
Resumen
El proceso de convergencia hacia el Espacio Europeo de Enseñanza Superior ha puesto de relieve la importancia del dominio de competencias transversales (CTs) durante la formación universitaria. Dichas competencias confieren al estudiante la capacidad de innovación y de adaptación a los cambios, siendo su adquisición necesaria para la vida profesional. En la Universidad Politécnica de Valencia, se han redactado 13 CTs que aúnan las competencias de la normativa vigente y las de las agencias de acreditación. En nuestro grupo de innovación educativa estudiamos diferentes métodos de enseñanza-aprendizaje y evaluación de las competencias transversales en asignaturas relacionadas con las ciencias de la vida. En concreto, en este trabajo presentamos una propuesta para evaluar la CT "Responsabilidad ética, medioambiental y profesional”. Esta competencia pretende la obtención de conocimientos, habilidades, destrezas y actitudes útiles para interactuar con el entorno, de forma ética, responsable y sostenible, ante uno mismo y los demás. Las asignaturas relacionadas con las ciencias de la vida y, en particular, sus créditos de laboratorio, resultan un marco idóneo para la adquisición de dicha competencia. Nuestra propuesta de evaluación de la misma se basa en una rúbrica que ha de ser cumplimentada por los pares a través de una aplicación telemática.
\end{abstract}

Palabras clave: competencia transversal, evaluación, e-rúbrica, laboratorio, responsabilidad ética, medioambiental y profesional

\section{Introducción}

La adaptación de las titulaciones al Espacio Europeo de Educación Superior (EEES) ha supuesto un gran cambio metodológico en el sistema de autonomía en el aprendizaje y en la

\section{(cc) EY-NC-ND 2015, Universitat Politècnica de València}


Evaluación de la competencia transversal "Responsabilidad ética, medioambiental y profesional” a través de una e-rúbrica en el laboratorio.

adquisición de competencias. Este proceso de cambio en la educación superior conduce implícitamente a nuevos procesos de evaluación donde los estudiantes adquieren un mayor protagonismo (Bartolomé, 2014). La participación del estudiante en el proceso de evaluación de manera activa se convierte en sí misma en un contenido del aprendizaje compartiendo responsabilidades, buscando consenso entre diferentes puntos de vista e intereses y cooperando en su elaboración (Bordas, 2001). Este modo de evaluación alcanza una finalidad formativa ya que el estudiante adquiere una mayor comprensión de la adquisición de las competencias y entiende el proceso de calificación. Según Somervell (1993) la evaluación participativa se ajusta a tres modelos: autoevaluación, el estudiante evalúa su propio trabajo, coevaluación, estudiante y docente asumen el rol de evaluadores y evaluación por pares, cada estudiante evalúa a un compañero (intra o intergrupo). En un contexto universitario donde los estudiantes tienen que desarrollar capacidades de pensamiento crítico durante el estudio de diferentes disciplinas, la evaluación por pares resulta un proceso activo, cooperativo y bidireccional. A la hora de implantar un modelo de evaluación de competencias, eje central del diseño curricular actual, hay que establecer los criterios de calidad que deben guiar cada una de las actividades evaluadoras: capacidad para adecuarse a los objetivos del proceso de aprendizaje, comparabilidad, reproducibilidad de las decisiones, transparencia y aceptabilidad, imparcialidad, autenticidad, complejidad cognitiva, significatividad y capacidad para la autoevaluación (Baartman, 2006).

En este trabajo se propone el uso de una rúbrica como instrumento para la evaluación por pares de competencias transversales en la educación superior. En concreto, se ha elaborado una matriz de criterios relacionados con objetivos de aprendizaje de la competencia transversal "Responsabilidad ética, medioambiental y profesional" para evaluar el nivel de rendimiento del estudiante. Ésta cumple las tres características fundamentales que exige una rúbrica: unos criterios de evaluación, una escala de valoración y una estrategia de calificación (Valverde, 2014). Además, con el fin de acercar la rúbrica al contexto tecnológico actual, se ha diseñado una e-rúbrica y se ha aplicado a los 120 estudiantes de la asignatura Química Biomolecular del Grado de Biotecnología de la Universidad Politécnica de Valencia matriculados durante el curso 2014-2015.

\section{Objetivos}

Este trabajo tiene como objetivo evaluar la competencia transversal "Responsabilidad ética, medioambiental y profesional” a través de una e-rúbrica cumplimentada por pares en el laboratorio. 


\section{Desarrollo de la innovación}

Nuestro grupo de innovación educativa, en colaboración con otros profesores del Departamento de Química, ha elaborado una e-rúbrica para evaluar la competencia transversal "Responsabilidad ética, medioambiental y profesional" en la asignatura Química Biomolecular, que se imparte en primer curso del grado en Biotecnología de la Universitat Politècnica de València.

La asignatura Química Biomolecular se estructura en dos bloques, en el primero "Química Orgánica" el objetivo principal es dar a conocer al alumno las bases teóricas y prácticas de la Química Orgánica como herramienta fundamental de trabajo y base sobre la que se sustenta la segunda parte de esta asignatura "Estructura y función de las biomoléculas". En esta segunda parte se ofrece una visión global de la estructura y función de las moléculas presentes en los seres vivos, lo que supone una panorámica general de la Bioquímica e incluso de la Biología Molecular, por cuanto se refiere también a las biomoléculas clave: proteínas y ácidos nucleicos.

La asignatura se imparte a un total de 120 estudiantes que se distribuyen en 2 grupos: uno de docencia en castellano (con 70 estudiantes) y otro denominado de Alto Rendimiento Académico (ARA) con docencia en inglés (con 50 alumnos). Las prácticas de laboratorio de la asignatura se realizan en grupo de como máximo 25 alumnos, dividiéndose el grupo de castellano en tres subgrupos (C1, C2 y C3) y el grupo ARA en dos (ARAI4 y ARAI5).

Del total de los 7,5 créditos de la asignatura, 2 son de prácticas de laboratorio. Dichos créditos de laboratorio se distribuyen en 6 prácticas, tres de ellas correspondientes al primer bloque (Química Orgánica) y tres al segundo (Bioquímica). La realización de las prácticas se lleva a cabo de dos en dos, manteniendo las mismas parejas en las tres primeras prácticas y cambiando de pareja en las tres últimas.

La innovación que se pretende desarrollar es la de evaluar la competencia transversal "Responsabilidad ética, medioambiental y profesional” mediante una e-rúbrica cumplimentada por pares en las sesiones de laboratorio de la asignatura Química Biomolecular.

Para ello, hemos desarrollado un cuestionario que evalúa a partes iguales la ética medioambiental y la ética de trabajo/profesional (Figura 1). La ética medioambiental se evalúa a través de 3 preguntas, con un $33.33 \%$ de peso cada una de ellas. La parte de la rúbrica correspondiente a la ética de trabajo/profesional, consta de 5 preguntas con un 20\% de peso cada una. Esta matriz de evaluación será cumplimentada telemáticamente por la pareja de laboratorio al finalizar cada una de las sesiones de prácticas de la asignatura. Así pues, cada alumno será evaluado durante las tres primeras prácticas por la misma persona y

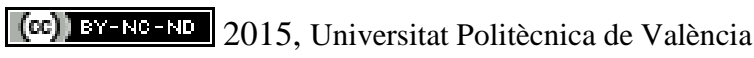

Congreso In-Red (2015) 
Evaluación de la competencia transversal "Responsabilidad ética, medioambiental y profesional" a través de una e-rúbrica en el laboratorio.

en las tres últimas por tres personas diferentes. En total, se dispondrá de 6 e-rúbricas para la evaluación de la competencia.

\section{Rúbrica para evaluar competencia transversal}

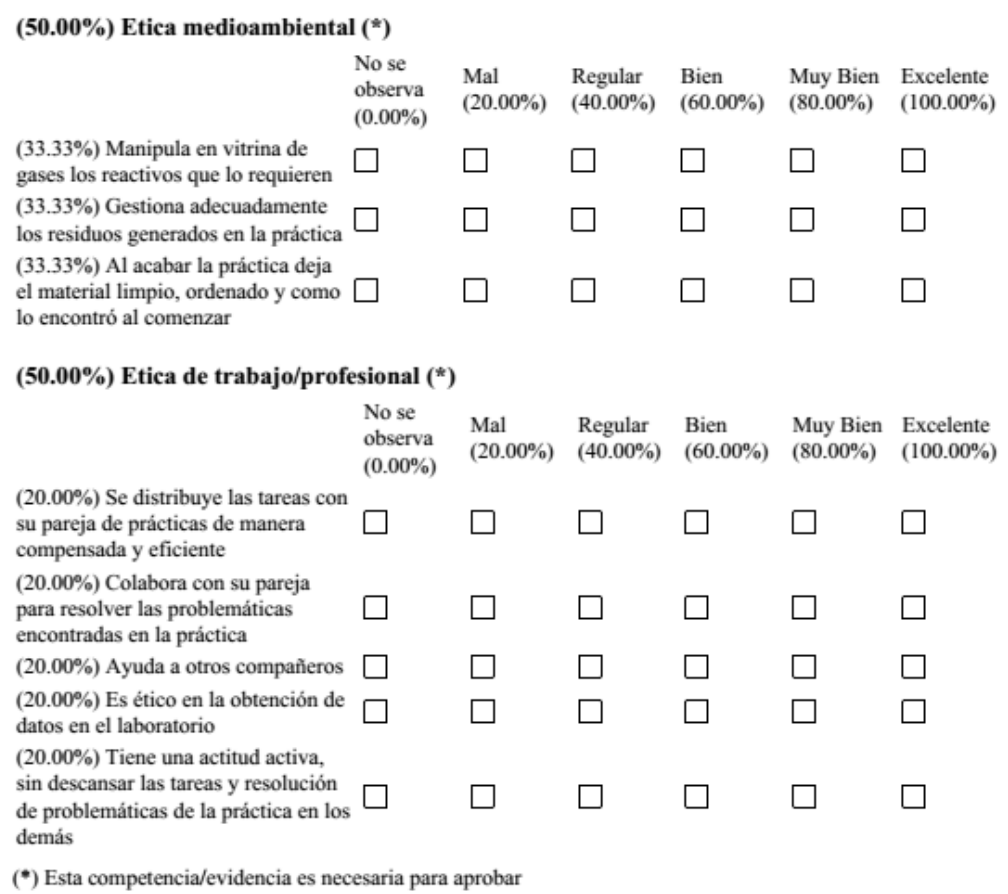

Fig. 1. Rúbrica para la evaluación por pares de la competencia transversal Responsabilidad ética, medioambiental y profesional

Con el fin de facilitar y motivar la cumplimentación de la rúbrica por parte del estudiante, se ha implementado una rúbrica on line utilizando la plataforma eRúbrica creada por el grupo Gtea (grupo de Investigación en Globalización, Tecnología, Educación y Aprendizaje de la Universidad de Málaga). El acceso a dicha herramienta es gratuito y libre, para los estudiantes y profesores de la Universitat Politècnica de València.

Las instrucciones para la cumplimentación de la e-rúbrica se facilitan al estudiante al inicio de la asignatura. Consisten en unirse a la rúbrica a través de un enlace y una contraseña para cada grupo de prácticas (C1, C2, C3, ARAI4 y ARAI5) que son proporcionadas por el docente. El acceso a la evaluación se realiza a través del portal https://gteavirtual.org/rubric/. 


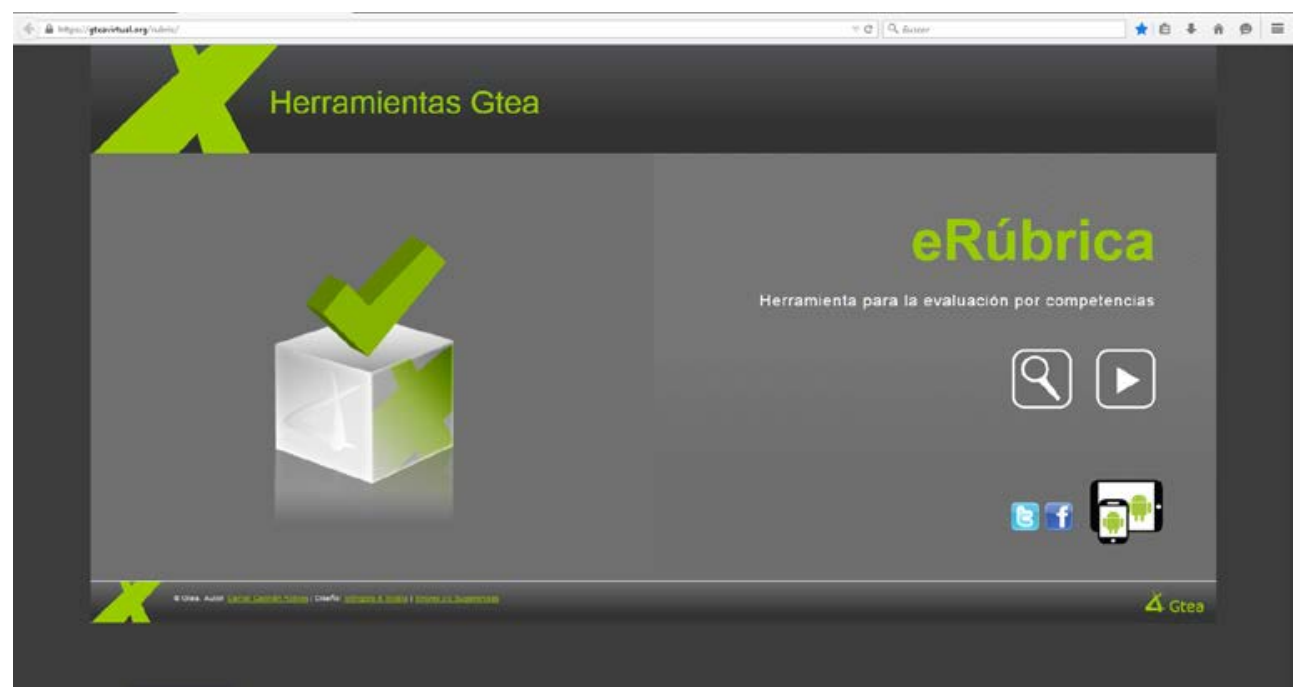

Fig.2. Impresión de pantalla del acceso al portal https://gteavirtual.org/rubric/ que permite la cumplimentación de la e-rúbrica

Una vez se accede, el estudiante selecciona la rúbrica correspondiente a su grupo de prácticas (Figura 3).

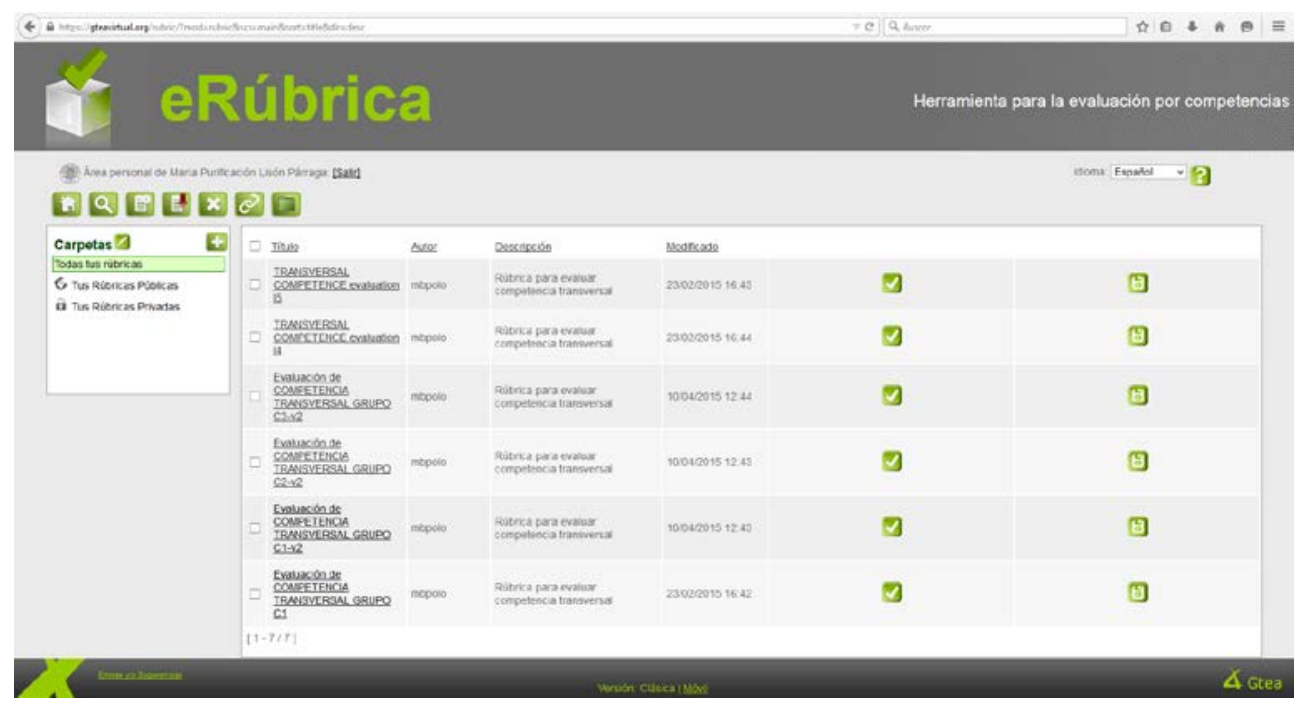

Fig. 3. Impresión de pantalla del acceso a cada una de las rúbricas desarrolladas para cada grupo de prácticas.

\section{(cc) EY-NC-ND 2015, Universitat Politècnica de València}

Congreso In-Red (2015) 
Evaluación de la competencia transversal "Responsabilidad ética, medioambiental y profesional” a través de una e-rúbrica en el laboratorio.

Por último, el estudiante selecciona a su pareja de prácticas y procede a su evaluación (Figura 4).

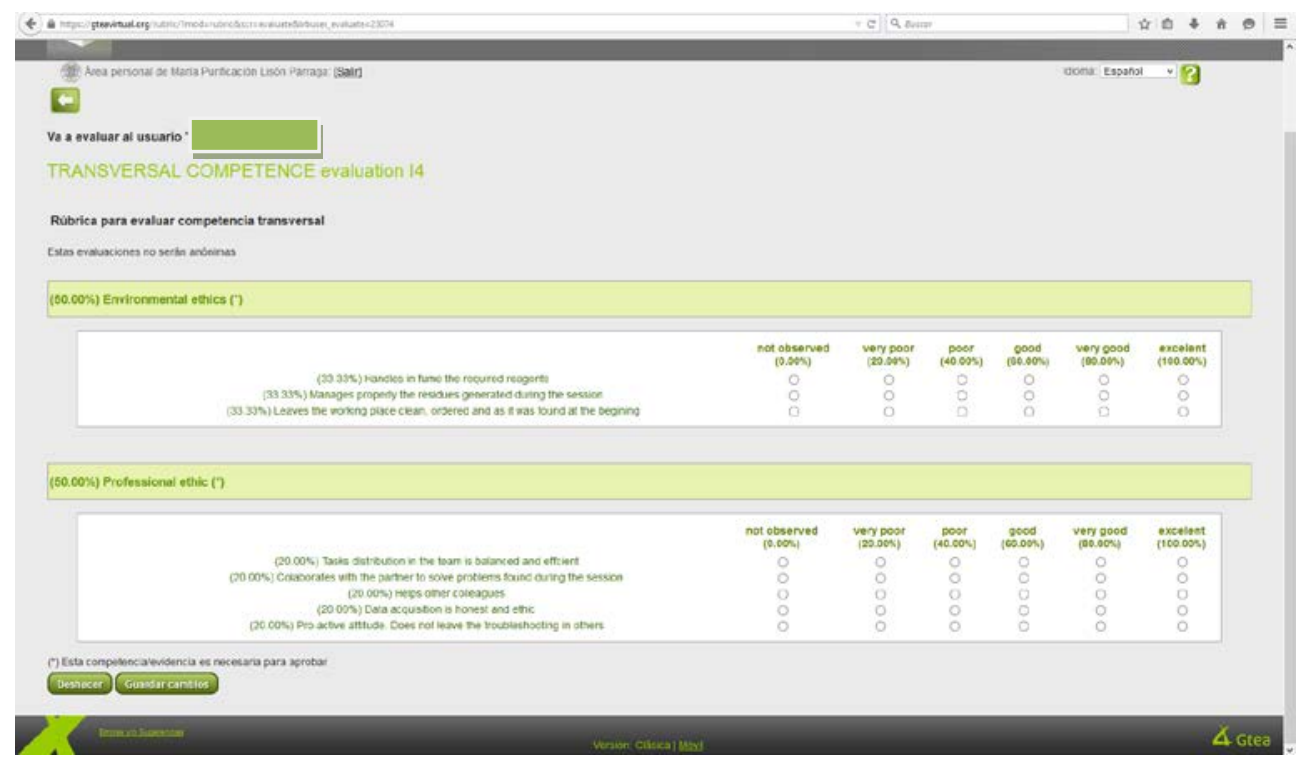

Fig 4. Impresión de pantalla de la e-rúbrica que los estudiantes han de cumplimentar (se muestra la del grupo de prácticas con docencia en inglés I4)

Una vez todos los estudiantes han realizado las 6 evaluaciones la aplicación permite importar los resultados a una página Excel para su posterior análisis.

\section{Resultados}

Se llevó a cabo el análisis de los resultados de la evaluación por pares de la competencia transversal "Responsabilidad ética, medioambiental y profesional" durante la realización de las prácticas de laboratorio, obtenidos a partir de las e-rúbricas cumplimentadas por 120 alumnos de la asignatura “Química Biomolecular” del curso 2014-2015.

Como se observa en la Figura 5, la nota global de la evaluación fue muy buena para todos los grupos (entre $8.9 \pm 0.86$ y $9.6 \pm 0.6$ ). Asimismo, los grupos que obtuvieron mejor calificación fueron el C1 y el ARAI4. Estos resultados sugieren que no existe un efecto grupo (castellano vs. ARA) en la forma de evaluación. 


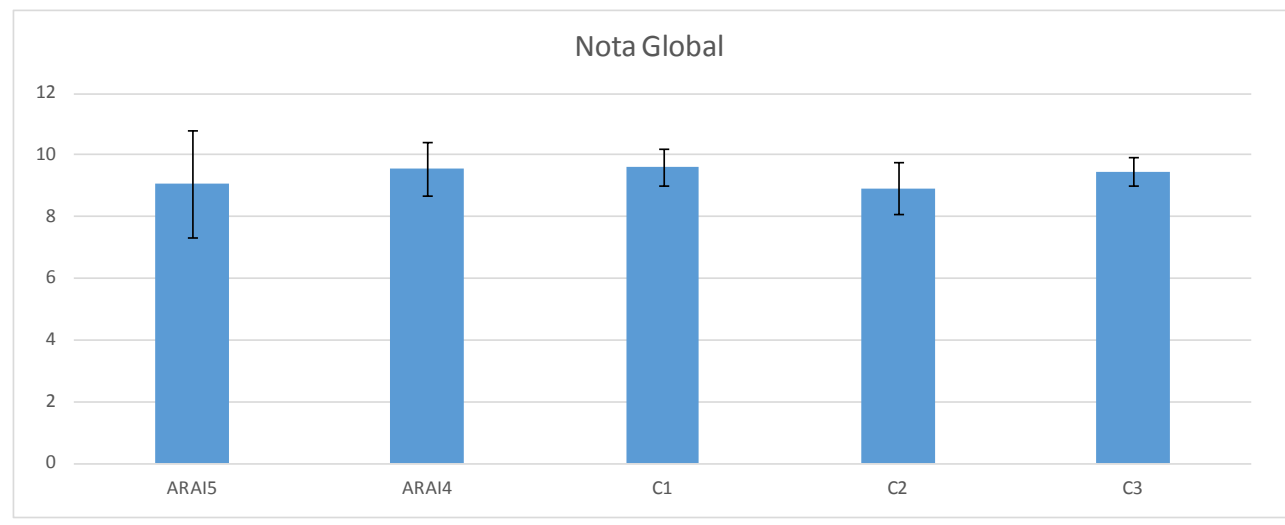

Fig 5. Nota media por grupo de la evaluación de la competencia transversal "Responsabilidad ética, medioambiental y profesional" obtenida mediante e-rúbrica cumplimentada por pares.

Con el fin de analizar los resultados correspondientes a las dimensiones competenciales "Ética medioambiental” y "Ética del trabajo" por separado, se analizaron los resultados de manera independiente. Como se puede apreciar en las Figuras 6 y 7, no se observan diferencias notables entre los resultados obtenidos para la evaluación de cada dimensión por separado y con respecto a la nota global, presentado perfiles similares en los tres casos. Tan sólo se observa cierta variación en los grupos ARAI4 y C1, que evalúan la "Ética del trabajo" con mejor y peor nota, respectivamente.

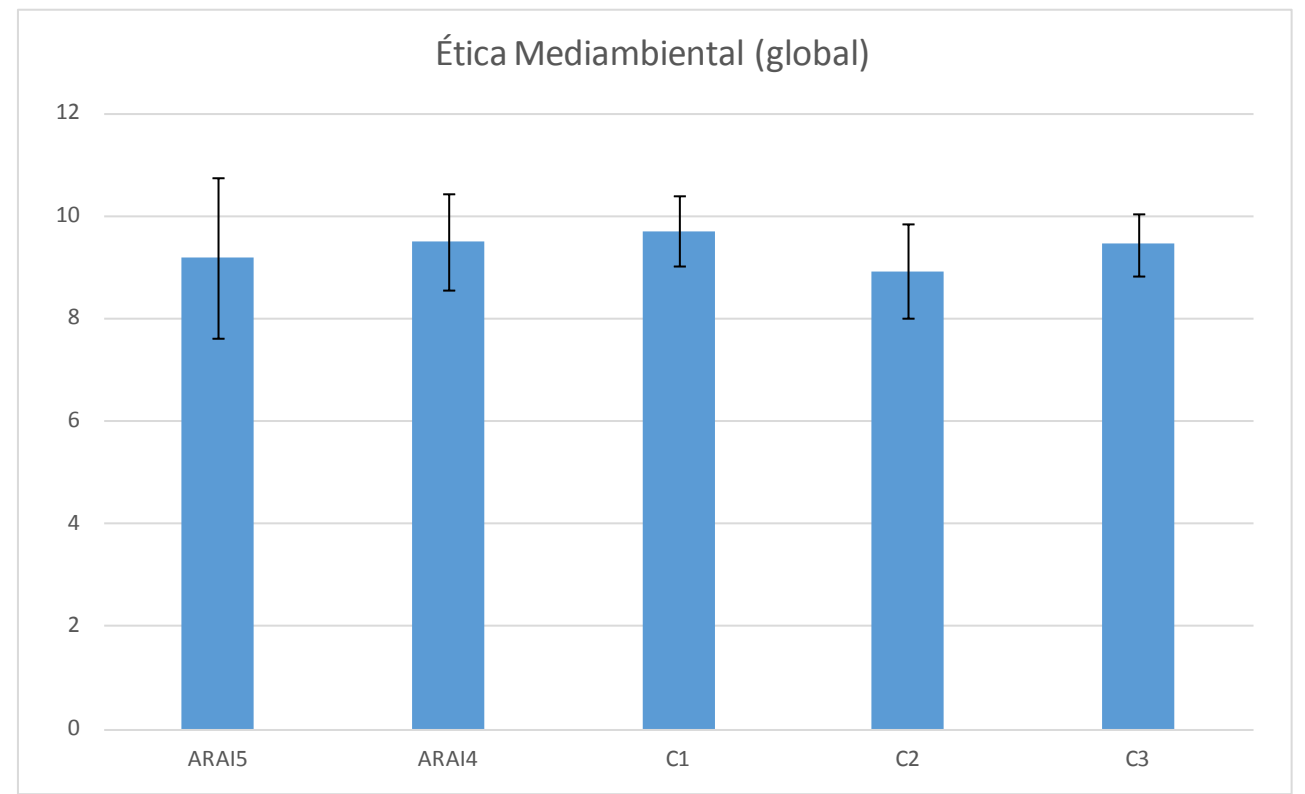

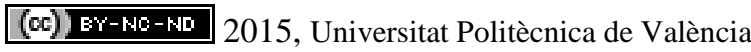

Congreso In-Red (2015) 
Evaluación de la competencia transversal "Responsabilidad ética, medioambiental y profesional” a través de una e-rúbrica en el laboratorio.

Fig 6. Nota media por grupo de la dimensión competencial "Ética medioambiental" evaluada mediante e-rúbrica cumplimentada por pares.

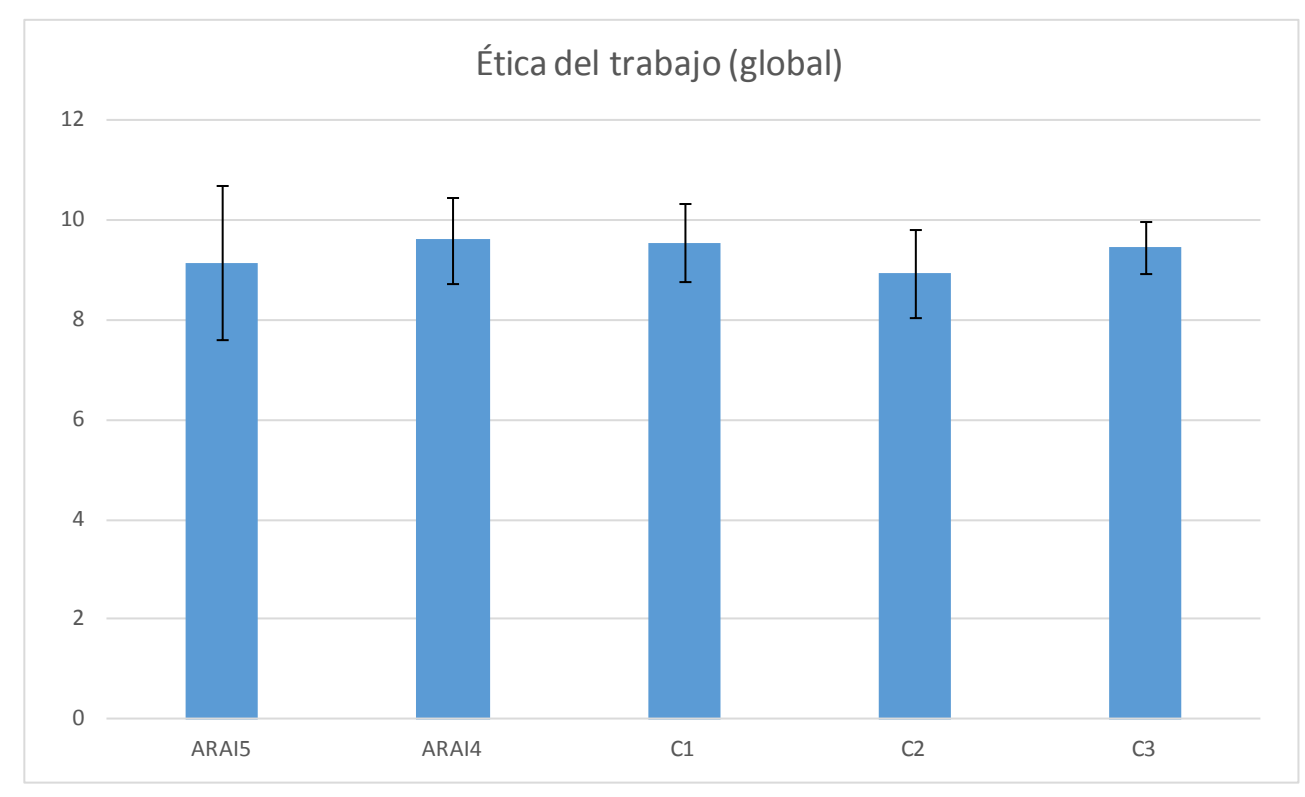

Fig 7. Nota media por grupo de la dimensión competencial "Ética del trabajo" evaluada mediante e-rúbrica cumplimentada por pares.

Por último, analizamos los resultados obtenidos para cada una de las preguntas (Figura 8). Dichos resultados nos permitieron detectar que las preguntas peor evaluadas fueron las correspodientes a gestión de los residuos (P1.2), distribución de tareas con su pareja (P2.1) y ayuda al resto de compañeros (P2.3). Estos resultados podrían indicar que se debería incidir en estos aspectos en cursos posteriores. Por otra parte, destaca la calificación media obtenida en la pregunta correspondiente a la recogida del material al acabar la práctica (P1.3). Efectivamente, los profesores podemos confirmar que dicha evaluación es correcta y acorde con la realidad, pues en todos los casos los alumnos dejan el material limpio y ordenado al acabar las sesiones de prácticas. 


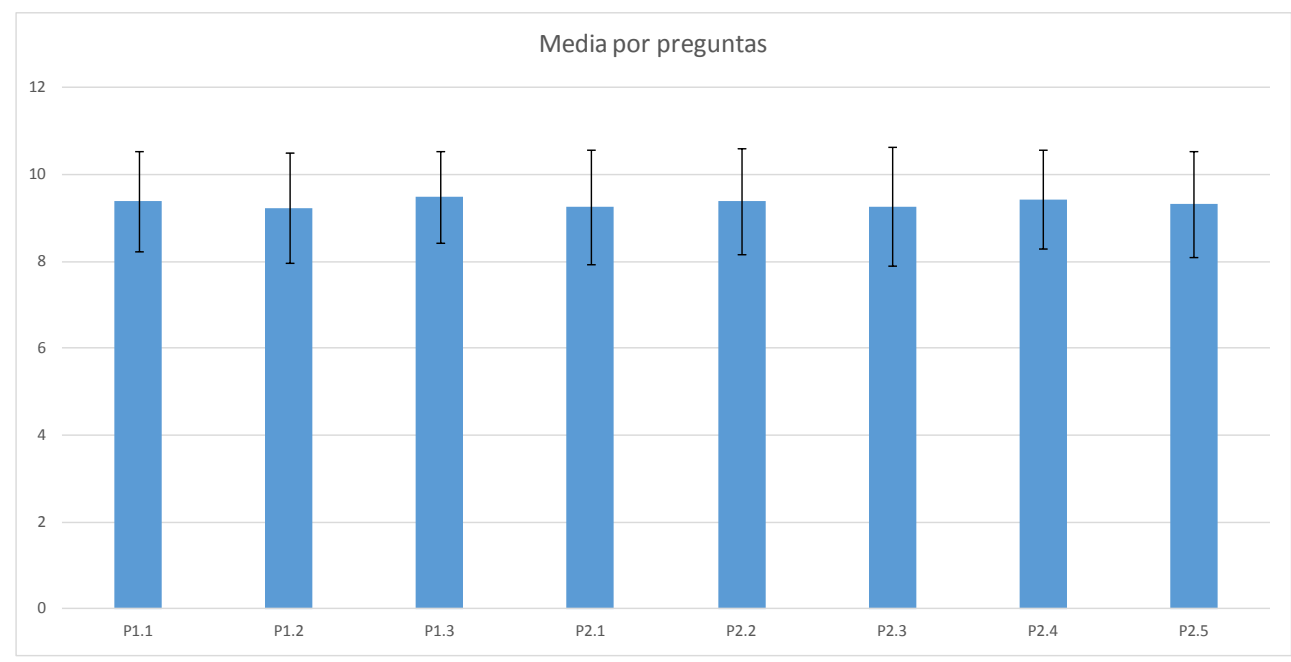

Fig 8. Nota media por pregunta de la evaluación de la competencia transversal "Responsabilidad ética, medioambiental y profesional" obtenida mediante e-rúbrica cumplimentada por pares.

En general, el grado de satisfacción de los profesores con esta herramienta de evaluación ha sido muy elevado. Hemos observado que la utilización de las e-rúbricas presenta una serie de ventajas, resultando ser más interactivas y dotando de una mejor autonomía a los estudiantes para conocer su estado de competencias adquiridas, y cuáles les queda aún por lograr en cualquier momento. Asimismo, nos ha permitido tener un mayor conocimiento para detectar competencias difíciles de alcanzar y disponer de un modo inmediato de mayor capacidad para la reedición y análisis de los resultados.

La puesta en práctica de la e-rúbrica nos ha permitido detectar algunos problemas (P) para los que proponemos las siguientes mejoras (M) que podrían implantarse en cursos posteriores:

1) (P) La plataforma puede resultar complicada de utilizar al principio, y requiere de una explicación práctica con instrucciones muy claras para que los alumnos sepan funcionar con ella desde la primera práctica.

(M) Se ha hecho un manual de instrucciones que será colgado en PoliformaT desde el primer día. Asimismo, pensamos que sería recomendable que fuera el mismo profesor (el más habituado a trabajar con la e-rúbrica) el que diera una explicación con el ordenador a todos los grupos.

(cc) EY-NC-ND 2015, Universitat Politècnica de València

Congreso In-Red (2015) 
Evaluación de la competencia transversal "Responsabilidad ética, medioambiental y profesional” a través de una e-rúbrica en el laboratorio.

2) (P) Ha habido retrasos en las evaluaciones tras cada práctica. Ello podría deberse a que no tengan claro el uso de la plataforma o a que lo dejen para cumplimentar en otro momento, lo que va en contra de la propia filosofía del método.

(M) Se facilitará la cumplimentación de la e-rúbrica en el horario de la sesión de prácticas (a su finalización) usando los móviles y se les dará un tiempo límite de 24h.

3) (P) Han surgido problemas cuando algún alumno cambiaba de grupo de prácticas pues no tenía acceso a la evaluación de su pareja desde su link del grupo.

(M) Se pretende crear una rúbrica para cada práctica en lugar de para grupo. Esto permitirá a su vez poder observar la progresión en la competencia a lo largo de las diferentes prácticas.

4) (P) Las elevadas calificaciones podrían deberse a que las evaluaciones no se realicen de forma seria, autónoma y objetiva.

(M) Se insistirá en la importancia en la adquisición de las comptencias transversales, así como en su correcta evaluación.

\section{Conclusiones}

Las asignaturas relacionadas con las ciencias de la vida y, en particular, sus créditos de laboratorio, pueden resultar un marco idóneo para la adquisición de la competencia transversal "Responsabilidad ética, medioambiental y profesional”.

La e-rúbrica cumplimentada por pares podría constituir un buen método para la evaluación de competencias transversales.

\section{Agradecimientos}

Este trabajo ha sido financiado por un Proyecto de Innovación y Mejora Educativa concedido por el Vicerrectorado de Estudios, Calidad y Acreditación de la Universitat Politècnica de València. 
Bañuls MJ et al.

\section{Referencias}

BAÑOS JE, PÉREZ J. (2005). New activities for developing generic skills in the health sciences.

BAARTMAN LKJ, BASTIAENS TJ, KIRSCHNER PA \& VAN DER VLEUTEN CPM (2006).The wheel of competency assessment: Presenting quality criteria for competency assessment programs. Studies in Educational Evaluation, 32(2), 153170.doi:10.1016/j.stueduc.2006.04.006

BARTOLOMÉ AR, MARTÍNEZ-FIGUEIRA PE, TELLADO-GONZÁLEZ F (2014) La evaluación del aprendizaje en red mediante blogs y rúbricas: ¿complementos o suplementos? Revista de docencia universitaria (Redu) Vol. 12 (1), Abril 2014, 159-176 ISSN: 1887-4592

BORDAS MI; CABRERA FA (2001). Estrategias de evaluación de los aprendizajes centrados en el proceso. Revista Española de Pedagogía, enero-abril, 218, 25-48.

DE MIGUEL DÍAZ M (2006). Metodologías de enseñanza y aprendizaje para el desarrollo de competencias. Orientaciones para el profesorado universitario ante el espacio europeo de educación superior. Madrid: Alianza editorial.

FORTEA MA (2009). Metodologías didácticas para la E/A de competencias. Miguel Angel Fortea. Formació professorat de la Unitat de Suport Educatiu (UJI) (Curso CEFIRE Castellón 2009: “Competencias en el ámbito de las ciencias experimentales. Programar y trabajar por competencias”)

KECHAGIAS K (2011).Teaching and Assessing Soft Skills. MASS Project.

LEIVA-BRONDO M, CEBOLLA-CORNEJO J, FERRIOL M, MERLE H, LÓPEZGRESA MP, LISÓN P, ESTERAS C, DÍEZ MJ, PICÓ B, PEIRÓ RM, PÉREZ-DECASTRO A (2014). Outcome Based Education In Life Science Subjects In Universitat Politècnica De València. International Association of Technology, Education and Development (IATED). INTED2014 Proceedings (978-84-616-8412-0)

NEALY C. (2005). Integrating soft skills through active learning in the management classroom. Journal of College Teaching and Learning, 2: 1-6.

PELLEGRINO JW, HILTON ML(2012). Education for life and work: Developing transferable knowledge and skills in the 21st century. The National Academies Press, Washintong D.C.

SOMERVELL H (1993). Issues in assessment, enterprise and Higher education: the case for self-peer and collaborative assessment.Assessment \& Evaluation in Higher Education, 18 (3), 221-233

(c) ) EY-NC-ND 2015, Universitat Politècnica de València

Congreso In-Red (2015) 
Evaluación de la competencia transversal "Responsabilidad ética, medioambiental y profesional” a través de una e-rúbrica en el laboratorio.

VALVERDE J, CIUDAD GÓMEZ A (2014). El uso de e-rúbricas para la evaluación de competencias en estudiantes universitarios. Estudio sobre fiabilidad del instrumento. Revista de docencia universitaria (Redu) Vol. 12 (1), Abril 2014, 49-79. ISSN: 1887-4592 